\title{
Ventricular coupling in single ventricle patients: a MRI study of cardiac biomechanics
}

\author{
Ramkumar Krishnamurthy ${ }^{1 *}$, Cory V Noel ${ }^{2}$, wei Pan ${ }^{1}$, Jeffrey Jacot ${ }^{3}$, Regina Lantin-Hermoso ${ }^{2}$, Rajesh Krishnamurthy ${ }^{1}$ \\ From 19th Annual SCMR Scientific Sessions \\ Los Angeles, CA, USA. 27-30 January 2016
}

\section{Background}

Ventricular dysfunction in patients with a single right ventricle (SRV) or a single left ventricle (SLV) is a known risk factor for morbidity and mortality. In normal hearts, LV and RV augment each other, while this is not possible in single ventricle (SV) patients. Ventricular-ventricular relationship in SV patients remain poorly understood, with only a few studies performed [1-3]. Our earlier results show a decreased peak circumferential $\left(\varepsilon_{\mathrm{cc}}\right)$ and longitudinal $\left(\varepsilon_{\mathrm{L}}\right)$ strain in SV patients when compared to a normal population [4]. However, an increase in longitudinal strain is noted in regions of decreased circumferential strain.

The purpose of this study is to understand the ventricular-ventricular interactions in systemic ventricles coupled and uncoupled to a dysfunctional ventricle.

\section{Methods}

We performed a prospective, IRB approved study of 24 subjects ( 9 normal age: $11.8+/-3 ; 8$ SRV age: $11.4+/$ 2.3; 7 SLV age: $12.7+/-4.2$ years). SLV and SRV patients were asymptomatic at time of imaging and were post total cavo pulmonary connection (TCPC).

Acquisition Protocol:

Strain information was acquired at three short axis slices at basal (coupled to dysfunctional ventricle), and apical (uncoupled) locations in all 18 subjects in a 1.5T MRI scanner (Philips Acheiva) using: a) Complementary Spatial Modulation of Magnetization (CSPAMM) ${ }^{4}$ images: $\varepsilon_{\mathrm{cc}}$; and b) Fast-Strain Encoded (fSENC $)^{5}$ images: $\varepsilon_{\mathrm{L}}$.

Data Analysis:

$\varepsilon_{\mathrm{cc}}$ and $\varepsilon_{\mathrm{L}}$ across all cardiac phases were calculated from SAX slices using Diagnosoft ${ }^{\mathrm{TM}}$. Global, free-wall and septal strain were calculated at both locations and ventricular coupling index $(\mathrm{VCI})$ is calculated as $\left(\varepsilon_{\mathrm{cc}}{ }^{*} \varepsilon_{\mathrm{L}} / 100\right)$.

\section{Results}

1) Strain values of SLV and SRV subjects demonstrate significant differences compared to normal subjects. (Figure 1)

2) Strain at the septal location is significantly reduced in single ventricle patients while the freewall strain is relatively normal.

3) Circumferential strain of the SV progressively reduces from the apex to the base, while the longitudinal strain increases.

4) VCI is significantly reduced at the basal septum pointing to the deleterious effect of the ventricular coupling between the systemic ventricle and the dysfunctional ventricle. Myocardial fiber arrangement and the hypoplastic chamber likely affect the regional differences demonstrated in this study (Figure 2).

\section{Conclusions}

Longitudinal strain is increased in regions where the circumferential strain is decreased in SV patients. However at the basal septum, where the dysfunctional ventricle is attached to the septum, it appears that the systemic ventricle mechanics is affected by deleterious ventricular coupling. Further studies are needed to understand differences between SRV and SLV cardiac biomechanics.

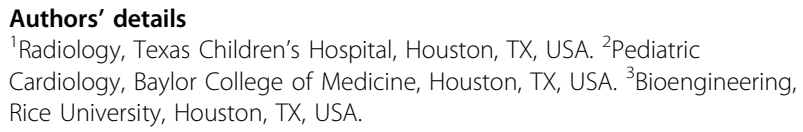

Published: 27 January 2016

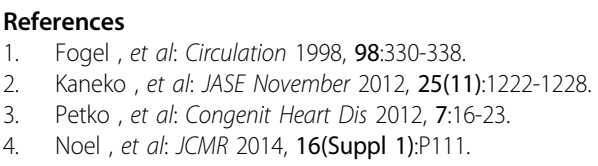

${ }^{1}$ Radiology, Texas Children's Hospital, Houston, TX, USA

Full list of author information is available at the end of the article 

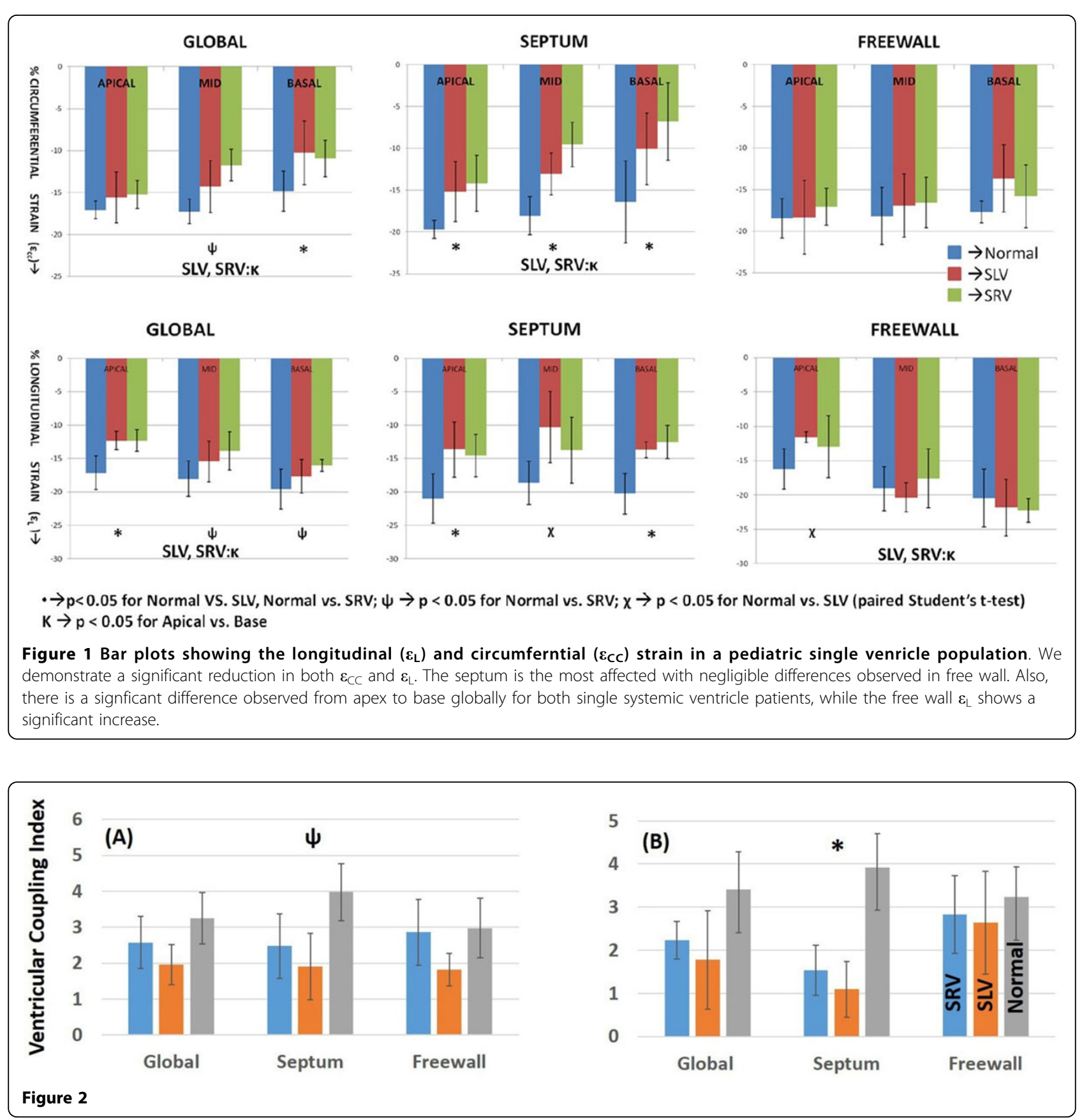

doi:10.1186/1532-429X-18-S1-P163

Cite this article as: Krishnamurthy et al:: Ventricular coupling in single ventricle patients: a MRI study of cardiac biomechanics. Journal of Cardiovascular Magnetic Resonance 2016 18(Suppl 1):P163.
Submit your next manuscript to BioMed Central and take full advantage of:

- Convenient online submission

- Thorough peer review

- No space constraints or color figure charges

- Immediate publication on acceptance

- Inclusion in PubMed, CAS, Scopus and Google Scholar

- Research which is freely available for redistribution 